\title{
基于约束构造算法的密码协议安全性分析 ${ }^{*}$
}

\section{李先贤 怀进鹏}

(北京航空航天大学计算机学院, 北京 100083)

摘要 基于形式化方法开发自动分析工具是密码协议安全性分析的一种有效 的方法, 然而, 由于密码协议参与主体的任意性、消息运算复杂性和运行的并发 性, 密码协议的安全性分析是高度计算复杂性的难题. 基于最近提出的密码协议 代数(CPA)模型, 采用代数方法描述密码协议活动, 精简密码协议描述, 提出一 个高效的密码协议安全性自动分析算法. 该算法通过泛多项式方程求解技术, 减 少密码协议安全性分析过程中产生的几余状态数量, 并可提供在无限状态空间 运行的协议安全性分析. 根据该算法, 实现了一个密码协议自动分析系统 ACT-SPA, 应用该系统分析了二十多个密码协议, 结果显示系统显著提高了运行 效率，并发现了新的密码协议攻击.

\section{关键词 密码协议 形式化分析 安全性验证}

随着大规模分布式应用的发展, 需要设计大量的密码协议(也称为安全协议) 解决日益增加的安全需求, 然而, 怎么知道所设计的密码协议是否满足相应的安 全需求呢? 这个问题极其复杂, 事实上, 许多原先声称的“安全”的密码协议后来发 现存在根本性的缺陷. Delov和Yao在 20 世纪 80 年代初首次应用形式化方法分析密 码协议的安全性 ${ }^{[1]}$, 这些年来已成为网络安全领域重要的研究方向和热点.

在近几年来关于密码协议安全性分析的研究中, 密码协议安全性分析主要 通过建立其形式化模型, 并开发相应的自动检验工具, 检验协议是否满足相应的 安全性目标. 这种方法的困难性主要由于无限密码协议运行状态引起的, 导致状 态的无限性包括以下 4 方面原因 ${ }^{[2]}$ :

- 参与协议运行的主体数量是任意的;

- 参与主体可执行协议任意多次; 
- 参与主体可同时参与任意多并发的协议会话;

- 协议相关的消息计算是无限的.

上面任何一种因素均可使密码协议的运行状态无限. 解决这个问题的一种 方法是限制以上几种可能引起无限状态的因素. 例如, 限制参与主体数量、协议 的运行步数和并发数, 以及限制消息组合数量等, 从而使密码协议的运行状态有 限, 然后, 通过自动检测工具对状态空间穷举搜索(通常需要优化搜索策略提高 算法效率), 这种方法通常称为模型检测. 现有许多的密码协议自动分析工具属 于这种类型, 利用模型检测工具已经发现了许多未知的安全协议攻击. 例如, 一 般性模型检测工具 $\mathrm{FDR}^{[3]}$ 和Mur $\phi^{[4]}$, 另外专用于密码协议分析的模型检测工具 Interrogator ${ }^{[5]}$ 和Brutus ${ }^{[6]}$ 等. 模型检测方法的一个显著优点是可以直接找出对密 码协议的攻击过程, 这种方法面临的主要问题有: 通常只能提供密码协议的不安 全性证明, 而不能证明密码协议的安全性. 这是由于模型检测只能在有限空间上 进行, 除非能够将密码协议的状态空间映射到一个有限空间(如文献[3]中提供的 协议分析), 这对一般情况是不可行的, 事实上已证明密码协议的安全性是不可

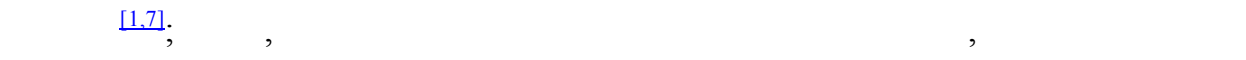
索方法通常面临状态数量爆炸问题.

分析密码协议安全性的另一种方法是定理证明方法, 与模型检测方法不同, 不是试图检测模型中的每一个状态, 而是试图证明安全目标公式在模型中的正 确性, 可用于分析密码协议在无限状态空间运行的安全性. 例如, NRL协议分析 器就是采用定理证明方法的密码协议分析工具 ${ }^{[8]}$, 以及基于Strand空间的Athena 分析算法采用符号状态表示和自由变量表示密码协议无限运行 ${ }^{[9]}$, 可分析由任意 参与主体和并发会话引起的无限状态下密码协议的安全性, 但Athena算法中仍限 制了攻击者的消息运算能力. 另外一些解决无限状态问题研究也采用了符号表 示方法, 例如, 文献[10]的研究. 然而, 在文献[10]中没有提供相应的分析工具.

本文的研究基于最近提出的一种密码协议形式化模型, 称为密码协议代数 (cryptographic protocol algebra, CPA)模型 ${ }^{[11]}$. 基于项重写系统和单向函数自由公 理建立消息代数通过引入 $\mathrm{CPA}$ 代数上的迁移函数概念刻画了接收消息与发送消 息之间的数学关系, 将协议运行过程描述为迁移函数序列, 用满足一定条件的序 列(称为正合序列)描述了攻击者可以完成的活动过程. 在文献[11]中还证明了可 以通过检验关于一些特定主体产生的正合序列验证安全协议在任意运行情况的 安全性, 但文献[11]没有提供协议的安全性自动分析算法.

本文的主要工作是根据 CPA 模型, 提出一种新的密码协议安全性自动分析 算法. 算法利用 CPA 模型中的迁移函数(可表示为泛多项式)对消息的约束关系排 除冗余状态. 算法未对在上面所提的 4 种引起无限状态空间因素进行限制, 因此 
可提供密码协议在无限运行状态下的安全性分析, 使结果更为可靠.

以下内容如下组织: 第 1 节简介 CPA 模型和密码协议紧致描述; 第 2 节提出 泛多项式方程及其求解算法; 第 3 节给出密码协议安全性分析基本算法, 并讨论 了算法的可靠性和完备性; 第 4 节描述一个新的密码协议安全性自动分析系统, 并给出实验数据; 第 5 节总结论文的工作.

\section{CPA 模型}

在这一节中我们简要介绍文献[11]中提出的密码协议代数模型—CPA 模型, 采用的符号和术语与文献[11]中一致.

\section{1 密码协议代数(CPA)}

消息项. 原子消息项集: PRIM $=\mathrm{ID} \cup \mathrm{KEY} \cup \mathrm{GEN}$ 是可数集, $\mathrm{GEN}=\bigcup_{a \in \mathrm{ID}} R_{a}$, 其中

- ID 是用于标记参与主体(包括攻击者)的名称集;

- KEY 是密钥集, 包括对称和非对称密钥, 每个密钥 $k$ 对应一个逆元 $k^{-1}$, 若 $k=k^{-1}$, 则 $k$ 为对称密钥; 若 $k \neq k^{-1}$, 则 $k$ 为非对称密钥;

- $R_{a}$ 是主体 $a$ 产生的消息集.

- 令 $X$ 是无限可数变量集, 变量有相应的类型.

密码协议运算. 密码协议运算是一些满足自由性公理的消息运算, 典型的包 括单向函数和字符联接运算. 主要包括:

- 字符联接运算“.””是二元运算 $x \cdot y$;

- 非对称密码运算 $\rho: \rho(x, k)$, 对称密码运算 $\mu: \mu(x, k)$;

- 一元运算 Hash 函数: $\operatorname{Hash}(x)$; 二元运算 MAC 函数: MAC $(x, k)$.

此外, 还可包含其他一些单向函数和相关运算. 协议 $P$ 的涉及运算的集合记 作 $\mathrm{F}_{P}$.

这些运算满足相应的代数等式, 例如, $(x \cdot y) \cdot z=x \cdot(y \cdot z), \mu\left(\mu(x, k), k^{-1}\right)=x$, $\rho\left(\rho(x, k), k^{-1}\right)=x$ 和 $\rho\left(\rho\left(x, k^{-1}\right), k\right)=x$ 等. 所有的等式集记作 $\mathrm{E}_{P}$.

由原子消息和协议运算可自然定义消息项，所有的消息项集合记作 $T(X)$, 所 有封闭项(不含变量的项，也称作基本消息)的集合记作 $\mathrm{M}$. 那么, $\left(T(X), \mathrm{F}_{P}\right)$ 构成一 个项代数, 而 $\left(M, F_{P}\right)$ 是它的一个子代数. 由运算及相应代数等式得到一个代数规 范: $A_{P}=\left(\mathrm{F}_{P}, \mathrm{E}_{P}\right)$. 利用项重写系统和自由性公理, 可引入密码协议相关其他消 息运算, 可以建立包含密码协议相关运算并满足密码自由性公理的代数称为密 码协议代数, 简称为 CPA(详见文献[11]).

多项式代数. 设 $A$ 是一个代数, $x_{1}, \cdots, x_{n}$ 是变量, 类似于环的多项式扩张, 可 定义代数 $A$ 的多项式扩张, $A\left[x_{1}, \cdots, x_{n}\right]$ 称为 $A$ 关于未定元 $\left\{x_{1}, \cdots, x_{n}\right\}$ 的多项式代 
数. $A\left[x_{1}, \cdots, x_{n}\right]$ 中的一个元称为一个 CPA 多项式, 本文中简称作多项式.

定义 1.1.1 若 $\sigma$ 是 $\mathrm{M}^{n} \cup\{\Delta\}$ 到 $M \cup\{\Delta, \perp\}$ 的一个映射, 并且当 $\vec{b}=$ $\left(b_{1}, \cdots, b_{n}\right) \in \mathrm{M}^{n} \cup\{\Delta\}, \sigma(\vec{b}) \in A\left[b_{1}, \cdots, b_{n}\right] \cup\{\Delta, \perp\}$, 则称 $\sigma$ 为代数 $A$ 上一个 $n$ 元 迁移函数. $A\left[x_{1}, \cdots, x_{n}\right]$ 上任何一个元可解释为 $A$ 上的一个 $n$ 元迁移函数, 这里符 号“ $\Delta$ ”表示空项, “ $”$ 表示终止符号, $\Delta$ 和 $\perp$ 不用于构成项.

设 $\sigma$ 为代数 $A$ 的一个 $n$ 元迁移函数, 规定 $\sigma$ 的定义域： $\operatorname{Dom}(\sigma)=$ $\left\{\vec{m} \in \mathrm{M}^{n} \cup\{\Delta\} \mid \sigma(\vec{m}) \neq \perp\right\}$.

定义 1.1.2 设 $\sigma$ 是代数 $A$ 的一个 $n$ 元迁移函数, 若存在项 $s$ 和 $t$ 使得 $\operatorname{Dom}(\sigma) \subseteq\left\{s\left(b_{1}, \cdots, b_{n}\right) \mid\left(b_{1}, \cdots, b_{n}\right) \in \mathrm{M}^{n}\right\}$, 并且 $\sigma\left(s\left(b_{1}, \cdots, b_{n}\right)\right)=t\left(b_{1}, \cdots, b_{n}\right)$, 则称 $\sigma$ 是表示的迁移函数, 其中符号 $s\left(b_{1}, \cdots, b_{n}\right)$ 表示用 $b_{1}, \cdots, b_{n}$ 替换 $s$ 的所有变量得到的 基本消息.

一个表示的迁移函数通常写作形式: $\langle\sigma, s, t>$. 本文主要讨论表示的迁移函 数, 后面叙述中, 若非特别说明, 迁移函数均指表示的迁移函数.

\section{2 密码协议的代数描述}

协议的基本消息交互描述 $\mathrm{CPA}$ 模型假设密码协议运行在开放环境下，参 与主体通过与环境消息交互实现安全通信，协议参与主体的消息接收和发送的 行为称为事件. 每个事件形式表示为 $\left\langle\sigma_{i j}(\alpha, l), m, m^{\prime}\right\rangle$ ，这里 $m$ 表示接收到的消息， $m^{\prime}=\sigma_{i, j}(\alpha, l)(m)$ 则表示发送的消息, $\sigma_{i, j}(\alpha, l)$ 是一个迁移函数, 其中 $(\alpha, l)$ 是标识 会话的参数, $\alpha=\left(a_{1}, \cdots, a_{N}\right)$ 表示 $N$ 方协议的 $N$ 个参与主体组成的向量, $l$ 是自然数 (详细定义见文献[11]).

$\sigma_{i, j}(\alpha, l)$ 称为事件函数. 密码协议 $P$ 的所有事件函数的集合记作 $\Sigma_{P}$, 称为事 件函数空间.

密码协议活动过程描述 在 CPA 模型中协议的执行过程体现为一系列的消 息交互，用满足一定条件的事件(或事件函数)序列表示. 相关的一些基本概念:

$1 ）$ 时序关系 $\preceq_{P} . \quad$ 对 $\forall \sigma_{i_{1} j_{1}}\left(\alpha_{1}, l_{1}\right) ， \quad \sigma_{i_{2} j_{2}}\left(\alpha_{2}, l_{2}\right) \in \Sigma_{P}$ ，规定 $\sigma_{i_{1} j_{1}}\left(\alpha_{1}, l_{1}\right) \preceq_{P}$ $\sigma_{i_{2} j_{2}}\left(\alpha_{2}, l_{2}\right)$ 当且仅当 $i_{1}=i_{2} \wedge \alpha_{1}=\alpha_{2} \wedge\left(l_{1}<l_{2} \vee\left(l_{1}=l_{2} \wedge j_{1} \leq j_{2}\right)\right)$. $\preceq_{P}$ 是偏序, 反 映了事件函数的先后顺序关系.

2) 迹. 直观地说, 就是不违反协议运行时序关系的事件函数序列. 形式表示 为: $\Sigma_{P}$ 上的一个有限序列 $\Gamma=\tau_{1} \tau_{2} \cdots \tau_{s}$, 若满足如下条件, 则称 $\Gamma$ 是协议的一条迹.

- 对任何 $1 \leq i \leq s$, 不存在 $\tau_{i}, \tau_{j}$ 使得 $\tau_{j} \preceq_{P} \tau_{i}$ ；

- 当 $\sigma_{i j}(\alpha, l) \in \Gamma$, 则对 $1 \leq k \leq j, \sigma_{i k}(\alpha, l) \in \Gamma$, 
其中每个事件函数 $\tau_{i}$ 称为迹 $\Gamma$ 的节点, 记作 $(\Gamma, i)$, 并称 $i$ 为节点 $(\Gamma, i)$ 在的 $\Gamma$ 中序号; 自然数 $s$ 称为迹 $\Gamma$ 的长度; 如果 $1 \leq i \leq j \leq s$, 称节点 $(\Gamma, i)$ 在 $\Gamma$ 中先于节点 $(\Gamma, j)$, 记 作 $(\Gamma, i) \operatorname{precedes}_{\Gamma}(\Gamma, j)$.

迹对应的事件序列称为迹序列, 形如 $\left\langle\tau_{1}, c_{1}, c_{1}^{\prime}\right\rangle\left\langle\tau_{2}, c_{2}, c_{2}^{\prime}\right\rangle \cdots\left\langle\tau_{n}, c_{n}, c_{n}^{\prime}\right\rangle$, 其中 $\tau_{i}$ 是事件函数, $c_{i}, c_{i}^{\prime}$ 是消息项. 不含变量的迹序列称为迹实例.

迹序列的保持顺序关系的子序列称为子迹序列，若 $\Lambda$ 是 $\Gamma$ 的子迹序列，记作 $\Lambda \sqsubseteq \Gamma$.

协议 $P$ 的所有迹序列集合记作 $\Sigma_{P}^{*}$ ，称为迹序列空间.

$3)$ 正则迹与角色. 设迹 $\sigma_{i, 1}(\alpha, l) \cdots \sigma_{i, n}(\alpha, l)$ 满足不存在 $k>n$ 使得 $\sigma_{i, k}(\alpha, l)$ $\in \Sigma_{P}$, 则称为协议 $P$ 的第 $i$ 个角色相关的正则迹, 记作 $\Sigma_{i}(\alpha, l)$. 正则迹 $\Sigma_{i}(\alpha, l)$ 描 述了协议 $P$ 的第 $i$ 个角色. 密码协议 $P$ 的所有正则迹的集合记作 $R_{P}$.

攻击者的描述. 在 CPA 模型中, 攻击者不是一个实体而是多个非诚实主体 集合, 与诚实主体的唯一区别在于拥有不同的初始知识, 在模型中并不需要专门 的形式概念描述攻击者, 只需刻画其初始知识集, 用 $D_{0}$ 表示, 称为攻击者初始代 数, 它是由一些初始消息生产的代数(由一个正则迹确定, 详见文献[11]).

4) 正合序列. 设 $\Gamma=\left\langle\tau_{1}, c_{1}, c_{1}^{\prime}\right\rangle\left\langle\tau_{2}, c_{2}, c_{2}^{\prime}\right\rangle \cdots\left\langle\tau_{n}, c_{n}, c_{n}^{\prime}\right\rangle$ 是包含一条正则迹 $\Sigma$ 的 迹序列, 若 $\Gamma$ 的一个节点 $\left\langle\tau_{i}, c_{i}, c_{i}^{\prime}\right\rangle$ 满足 $c_{i} \in D_{0}\left[c_{1}, \cdots, c_{i-1}\right]$, 其中 $D_{0}$ 是相应攻击者 初始知识代数, 则称 $\left\langle\tau_{i}, c_{i}, c_{i}^{\prime}\right\rangle$ 为 $\Gamma$ 的一个正合 $\Sigma$-节点; 若序列 $\Gamma$ 的每个节点均是 正合 $\Sigma$-节点, 则称 $\Gamma$ 是正合 $\Sigma$-序列(在上下文含义明显时, 正合 $\Sigma$-序列简称为正合 序列).

用 $D_{0}[\Gamma]$ 表示 $D_{0}\left[c_{1}^{\prime}, c_{2}^{\prime}, \cdots, c_{n}^{\prime}\right], \operatorname{Ext}(\Sigma)$ 表示所有正合 $\Sigma$-序列的集合.

符号. 设 $\Gamma$ 是迹序列, $\Sigma$ 是正则迹, $s$ 是一个消息, 如果 $s \in D_{0}[\Gamma]$, 则称 $s$ 关于 $\Sigma$ 属于 $\Gamma$, 记作 $s \in \in_{\Sigma} \Gamma$, 其中 $D_{0}$ 是由 $\Sigma$ 确定初始代数.

在 CPA 模型中, 用正合序列刻画密码协议可实现的完整执行过程, 即可表示 协议的正常运行过程, 也可表示针对协议的攻击活动过程.

5) 忠实节点和正规序列. 设 $\alpha=\left(a_{1}, \cdots, a_{N}\right)$ 是关于 $N$ 方协议的主体向量, $\left\langle\sigma_{j k}(\beta, l), s, t\right\rangle$ 是某个迹的节点, 若有 $(\beta)_{j} \in\left\{a_{1}, \cdots, a_{N}\right\}$, 则称为忠实的 $\alpha$-节点, 符 号 $(\beta)_{j}$ 表示 $\beta$ 的第 $j$ 个分量. 若迹序列 $\Gamma$ 是正合的 $\Sigma_{i}(\alpha, l)$-序列, 并且 $\Gamma$ 的每个节点 均为忠实的 $\alpha$-节点, 则称 $\Gamma$ 为正规的 $\Sigma_{i}(\alpha, l)$-序列.

用 $\operatorname{Nor}(\Sigma)$ 表示所有正规 $\Sigma$-序列的集合.

\section{3 密码协议安全性模型}

密码协议安全性逻辑. 语法: 项的构成包括: 包括消息常量 $\{a, b, \cdots\}$, 迹序 
列常量 $\{\Gamma, \Sigma, \Lambda, \cdots\}$ 和迹序列变量 $\{X, Y, \cdots\}$. 谓词符号包括: ᄃ, EXT 和 $\epsilon$.

原子公式: $\Sigma \sqsubseteq X, \operatorname{EXT}(\Sigma, X)$ 和 $(a, \Sigma) \epsilon X$;

若 $f_{1}, f_{2}$ 是命题公式, 则 $\neg f_{1}, f_{1} \wedge f_{2}$ 和 $\forall X f_{1}$ 也是命题公式.

其中 $a$ 是消息常量, $\Sigma$ 是迹序列常量, $X$ 是迹序列变量, 而 $f_{1}$ 只含自由变量 $X$. 可自然引入其他的逻辑运算符: $\Rightarrow, \vee$ 和 $\exists$ 等.

语义: 关于协议 $P$ 的模型是五元组 $M_{P}=\left(\mathrm{M}, \Sigma_{p}^{*}, R_{P}, E_{P}, \Phi\right)$, 其中 $\mathrm{M}$ 是基本 消息代数, $\Sigma_{p}^{*}$ 是迹序列空间, $R_{P}$ 是 $\Sigma_{p}^{*}$ 中的正则迹序列集合, $E_{P}=\bigcup_{\Sigma \in R_{P}} \operatorname{Ext}(\Sigma)$, 其 中 $\operatorname{Ext}(\Sigma)$ 表示所有正合的 $\Sigma$-序列集合, $\Phi$ 是对常量和变量的解释. 语义解释如下:

$\Phi(a) \in \mathrm{M}, \Phi(\Sigma) \in \Sigma_{p}^{*}$, 而 $\Phi(X)$ 解释为变量到 $\Sigma_{p}^{*}$ 上的映射;

$M_{P} \mid=\Sigma \sqsubseteq X$ 当且仅当 $\Phi(\Sigma) \sqsubseteq \Phi(X)$;

$M_{P} \mid=\operatorname{EXT}(\Sigma, X)$ 当且仅当 $\Phi(\Sigma) \in R_{P}$ 且 $\Phi(X) \in \operatorname{Ext}(\Phi(\Sigma))$;

$M_{P} \mid=(a, \Sigma) \in X$ 当且仅当 $\Phi(\Sigma) \in R_{P}$ 且 $\Phi(a) \in_{\Phi(\Sigma)} \Phi(X)$.

$(a, \Sigma) \epsilon X$ 也可简略地记作 $a \epsilon_{\Sigma} X$, 对逻辑联接词 $\neg, \wedge$ 和量词 $\forall$ 的公式语义解 释是自然的.

协议的安全性规范. 在 CPA 模型中, 通过检验正合序列是否满足相应的安全 性目标来验证密码协议的安全性, 本文我们主要讨论两类安全性: 秘密性和认证 性(包括数据源认证和实体认证).

1) 秘密性. 设 $\Gamma$ 是一个正合的 $\Sigma$-序列, 我们说 $\Gamma$ 对消息 $s$ 保密, 意味着攻击 者不能从该序列的输出消息计算出 $s$, 即 $\neg a \epsilon_{\Sigma} \Gamma$. 协议秘密性用

$$
\forall X(\operatorname{EXT}(\Sigma, X) \wedge \Sigma \sqsubseteq X) \Rightarrow \neg s \epsilon_{\Sigma} X \text { 简写为 } \Sigma \text { Secret } s
$$

表示, 称公式 $\Sigma$ Secret $s$ 为协议的秘密性安全目标, 其中 $s$ 是由 $\Sigma$ 确定的消息项.

2) 认证性. 可认证性解释为一种对应性. 可用

$$
\forall X(\operatorname{EXT}(\Sigma, X) \wedge \Sigma \sqsubseteq X) \Rightarrow \Lambda \sqsubseteq X \text { 简写为 } \Sigma \text { Corresp } \Lambda,
$$

规范. 该公式称为协议的认证性安全目标, 其中 $\Lambda$ 是由 $\Sigma$ 确定的迹序列.

\section{4 密码协议分析紧致模型}

协议安全性目标规范和约简. 按照上面的分析逻辑, 判断协议的安全性, 需 要验证所有安全性目标公式的正确性, 而每个正则迹有相应的一个安全目标公式, 因此需要对安全目标规范和约简. 实际上, 表示协议安全目标的公式是由角色以 及相应的参数确定的, 可以对角色正则迹进行变量替换得到. 形式化表述如下:

$P$ 是一个 $N$ 方密码协议, $\Sigma$ 是正则迹, 相应的安全目标为 $\Sigma$ Secret $s$ (或 
$\Sigma$ Corresp $\Lambda), \phi$ 是一变量替换, 则 $\phi(\Sigma)$ 相应的安全目标为 $\phi(\Sigma)$ Secret $\phi(s)$ (或 $\phi(\Sigma)$ Corresp $\phi(\Lambda))$.

注 1.4.1 变量替换 $\phi:\left\{t_{1} / x_{1}, \cdots, t_{n} / x_{n}\right\}$ 是一个映射, $\phi(t)=t\left\{t_{1} / x_{1}, \cdots, t_{n} / x_{n}\right\}$ 表示 用 $t_{i}$ 替代变量 $x_{i}$ 在 $t$ 中的所在出现得到的项 ${ }^{[11]}$.

设 $\Sigma$ Secret $s$ 或 $\Sigma$ Corresp $\Lambda$ 是协议 $P$ 的安全目标, 我们规定:

- $P \mid=\Sigma$ Secret $s$ 当且仅当对任何变量替换 $\phi$ 有 $M_{P} \mid=\phi(\Sigma)$ Secret $\phi(s)$;

- $P \mid=\Sigma$ Corresp $\Lambda$ 当且仅当对任何变量替换 $\phi$ 有 $M_{P} \mid=\phi(\Sigma)$ Corresp $\phi(\Lambda)$.

由文献[11]的结果：可以通过验证固定的一组主体与攻击者参与产生的迹序 列安全性来分析一个协议的安全性, 即有下面引理.

引理 1.4.1 $N$ 方协议 $P$ 是安全的当且仅当 $P \mid=\Sigma_{i}(\alpha, l)$ Secret $s_{i}$ (或 $P \mid=$ $\Sigma_{i}(\alpha, l)$ Corresp $\left.\Lambda_{i}\right), i=1, \cdots, N$, 其中 $\alpha$ 是一组固定主体.

这个引理意义在于可以排除大量参与主体产生的正则迹序列相应的安全性 目标公式，只需验证有限几个安全性目标公式的正确性，从而为建立高效协议安 全性分析算法建立基础.

迹序列空间约简. 由文献[11]和引理 1.4.1 只需验证协议的正规序列是否满足 相应几个安全目标即可, 但正规序列集合仍可能是无限的, 需要进一步规范.

设 $\alpha=\left(a_{1}, \cdots, a_{N}\right)$ 是诚实主体向量, $p_{1}, \cdots, p_{N-1}$ 是 ID 型变量, $A=\left\{a_{1}, \cdots, a_{N}, p_{1}\right.$, $\left.\cdots, p_{N-1}\right\}$, 令集合

$\mathrm{NOR}_{P}=\left\{\sigma_{i j}(\beta, l) \mid \sigma_{i j}(\beta, l)\right.$ 是忠实的 $\alpha$-节点并且 $\left.\alpha \subseteq A, 1 \leq i \leq N, 1 \leq j \leq l_{i}\right\}$,

其中 $l_{i}$ 是协议 $P$ 的第 $i$ 个角色正则迹的长度, $\mathrm{NOR}_{P}$ 称为主节点集.

那么, 任何一个忠实的 $\alpha$-节点均可写成形式 $\phi\left(\left\langle\sigma_{i j}(\beta, l), s, t\right\rangle\right)$, 其中 $\phi$ 是变量 替换, 而 $\left\langle\sigma_{i j}(\beta, l), s, t\right\rangle \in \mathrm{NOR}_{P}$. 我们知道正规序列由忠实节点组成, 因此可以通 过有限符号集 $\mathrm{NOR}_{P}$ 和变量替换表示所有的正规序列. 这里没有对参与者数量、 运行的并发数、协议执行次数以及消息运算长度做人为的限制, 即约简后的模型 反映了协议在无限空间运行的性质.

\section{2 泛多项式方程及其算法}

\section{1 消息代数计算}

本节的目的是基于 CPA 代数建立消息计算算法. 为了便于算法理解, 这里将 代数运算表示为消息项推导规则. 假设密码协议仅涉及以下几类运算, 若还有其 他运算，增加规则即可，而不影响算法.

消息项推导规则. 由 5 个合成规则和 5 个消解规则组成, 分别对应相应的密 码协议运算: 
合成规则: $\frac{m_{1}, m_{2}}{m_{1} \cdot m_{2}}(\bullet) ; \frac{a, k}{\rho(a, k)}(\rho) ; \frac{a, k}{\mu(a, k)}(\mu) ; \frac{a, k}{\operatorname{MAC}(a, k)} \quad$ (MAC);

$$
\frac{a}{\operatorname{Hash}(a)}(\text { Hash) }
$$

消解规则: $\frac{m_{1} \cdot m_{2}}{m_{1}}\left(\pi_{1}\right) ; \frac{m_{1} \cdot m_{2}}{m_{2}}\left(\pi_{2}\right) ; \frac{\rho(a, k), k^{-1}}{a}\left(\rho_{1}\right) ; \frac{\rho\left(a, k^{-1}\right), k}{a}\left(\rho_{2}\right)$; $\frac{\mu(a, k), k}{a}\left(\mu^{-1}\right)$.

定义 2.1.1 设 $T$ 是由上面规则构成的推导树, 如果 $T$ 的叶节点集为 $\left\{a_{1}, \cdots, a_{n}\right\}$, 根节点为 $a$, 则称 $a$ 由 $\left\{a_{1}, \cdots, a_{n}\right\}$ 经 $T$ 导出, 记作 $\left\{a_{1}, \cdots, a_{n}\right\} \mid-_{T} a$.

设 $A$ 是一个消息项集, 对消息项 $a$, 若存在一个推导树 $T$ 和 $A$ 的有限子集 $\left\{a_{1}, \cdots, a_{n}\right\}$ 使 $\left\{a_{1}, \cdots, a_{n}\right\} \mid-_{T} a$, 则称 $a$ 由 $A$ 导出, 记作 $A \mid-a$. 若消息项集 $B$ 的每个 元都可由 $A$ 导出, 则称 $B$ 可由 $A$ 导出, 记作 $A \mid-B$.

设 $A$ 是消息项集, 若 $A$ 在导出关系“|-”下封闭, 且满足当 $k^{-1} \in A \cap \mathrm{KEY}$ 有 $k \in A$, 则称 $A$ 为一个消息闭包. $C$ 是一些消息集, 包含 $C$ 的最小的消息闭包, 记 作 $\mathscr{L}(C)$. 若 $C$ 是基本消息集, 则 $\mathscr{L}(C)$ 就是是由 $C$ 生成的 $\mathrm{CPA}$ 代数.

定义 2.1.2(子项完全集) 设 $t$ 是一个消息项, $t$ 的一个子项完全集是如下递归 定义的一些 $t$ 的子项集合:

1) 若 $t$ 是原子消息，则 $t$ 的子项完全集是 $\{t\}$;

2) 设 $U_{1}$ 和 $U_{2}$ 分别是 $t_{1}$ 和 $t_{2}$ 的子项完全集, 若 $t=t_{1} \cdot t_{2}$, 则 $\{t\}$ 或 $U_{1} \cup U_{2}$ 是 $t$ 的 子项完全集; 若 $t=\operatorname{Hash}\left(t_{1}\right)$, 则 $\{t\}$ 或 $U_{1}$ 是 $t$ 的子项完全集;

$3)$ 若 $t=\mu\left(t_{1}, k\right), t=\rho\left(t_{1}, k\right)$ 或 $t=\operatorname{MAC}\left(t_{1}, k\right)$, 则 $\{t\}$ 或 $U_{1} \cup\{k\}$ 是 $t$ 的子项完全 集, 其中 $U_{1}$ 是 $t_{1}$ 的子项完全集.

显然, 若 $U$ 是消息项 $m$ 的一个子项完全集，则 $U \mid-m$.

定义 2.1.3 设 $I \subseteq T(X)$ 是一些消息项集, 若对 $\forall g \in I, a \in T(X), k$ 满足

1) $a \cdot g, g \cdot a \in I$; 且

2) $\rho(g, k) \in I$ 和 $\mu(g, k) \in I$,

则称 $I$ 是一个理想 ${ }^{1)}$.

设 $G$ 是一些消息项集, $I(G)$ 表示包含了 $G$ 的最小理想, 称为 $G$ 生成的理想. 特 别地, $g$ 是基本消息, $I(g)$ 表示 $g$ 生成理想. $I(G)=\bigcup_{g \in G} I(g)$.

定义 2.1.4 若 $a \in I(g)$, 则称 $g$ 是 $a$ 的一个内项, 记作 $g \unlhd a$.

容易验证关系 $\unlhd$ 是自反的、传递的和反对称的.

1) 理想的概念源于代数中环论, 文献[12]首次引入消息代数计算 
合一 (unifier). $s, t$ 是两个消息项, $\phi$ 是一个变量替换, 若 $\phi(s)=\phi(t)$, 称 $\phi$ 是 $s, t$ 的一个合一.

最一般合一(most general unifier, MGU). 设 $\phi$ 是 $s, t$ 的一个合一, 若对任何另 一个合一 $\sigma$, 存在一个变量替换 $\theta$, 使 $\sigma=\theta \circ \phi$, 则称 $\phi$ 是 $s, t$ 的一个最一般的合一, 简称 $\mathrm{MGU}^{1)}$.

定义 2.1.5 设 $s \unlhd s^{\prime}$, 若 $\phi$ 是 $s^{\prime}$ 和 $t$ 的一个 MGU，则称 $\phi$ 是 $s$ 和 $t$ 的一个 $\triangleleft$ $-\mathrm{MGU}$.

定义 2.1.6 设 $g$ 是 $a$ 的一个内项, $K$ 是密钥项集, 若满足 $K \cup\{a\} \mid-g$, 则称 $g$ 是 $a$ 的一个 $K$-可逆内项, 记作 $g \unlhd_{K} a$.

定理 2.1.1 设 $A$ 是消息项集，令 $K$ 是包含于 $\mathscr{L}(A)$ 中的密钥项集，那么，对任 何消息项 $m, m \in \mathscr{L}(A)$ 当且仅当存在 $m$ 的一个子项完全集 $U_{m}$, 使得对 $\forall c \in U_{m}$, 存在 $a \in A$ 满足 $c \unlhd_{K} a$.

证 “ $\Leftarrow ”$. 注意到 $A \mid-K$, 由条件容易得到 $A \mid-U_{m}$ 和 $U_{m} \mid-m$, 故 $A \mid-m$, 即 $m \in \mathscr{L}(A)$.

“ $\Rightarrow ”$. 为简化叙述，我们规定一个概念. 设 $A, K$ 是如上所述集合，若消息集 $B$ 满足对 $\forall b \in B$, 存在 $a \in A$ 使得 $b$ 是 $a$ 的一个 $K$-可逆内项, 则称 $B$ 内包含于 $A$, 记 作 $B \subseteq$ IN $A$.

那么, 定理可叙述为 $m \in \mathscr{L}(A)$ 当且仅当存在 $m$ 的一个子项完全集 $U_{m}$ 内包含 于 $A$.

设 $m \in \mathscr{L}(A)$ 即 $A \mid-m$, 存在 $A$ 的有限子集 $A_{1}$ 和推导树 $T$, 使 $A_{1} \mid-_{T} m$.

我们对推导树 $T$ 的高度归纳证明结论. 当 $T$ 的高度为 0 , 即 $m \in A_{1}$, 结论显然 成立.

设 $T$ 的高度大于 0 , 根据 $T$ 的根节点 $m$ 关联的规则分别讨论.

1) 若 $T$ 的根节点关联的规则是一个消解规则 $\left(\pi_{1}, \pi_{2}, \rho_{1}, \rho_{2}\right.$ 或 $\left.\mu^{-1}\right)$, 这里我 们仅讨论 $\rho_{1}$, 对于其他 4 种情形可类似证明. 那么存在 $k \in \mathrm{KEY}$ 使得

$$
\left\{\rho(m, k), k^{-1}\right\}\left|-{ }_{q} m, A_{1}\right|-{ }_{T_{1}} \rho(m, k) \text { 和 } A_{1} \mid-k^{-1},
$$

其中 $T_{1}$ 是 $T$ 的高度小于 $T$ 的子树. 由归纳假设存在 $\rho(m, k)$ 的一个子项完全集 $S \subseteq \mathrm{IN}$ $A$. 由定义有 $S=\{\rho(m, k)\}$ 或 $S=U_{m} \cup\{k\}$, 其中 $U_{m}$ 是 $m$ 的一个子项完全集. 因此 当 $S=U_{m} \cup\{k\}$, 由 $U_{m} \subseteq S$ 和 $S \subseteq \subseteq_{\mathrm{IN}} A$, 立即得到 $U_{m} \subseteq \mathrm{IN} A$.

当 $S=\{\rho(m, k)\} \subseteq_{\mathrm{IN}} A$, 即存在 $a \in A$ 满足 $\rho(m, k) \unlhd_{K} a$, 但 $m \unlhd \rho(m, k)$, 由传递

1) 求两个项的最一般合一算法是“归结推导”的一个基本算法 
性即得 $m \unlhd a$. 另一方面, $A_{1} \mid-k^{-1}$ 故 $k^{-1} \in \mathscr{L}(A) \cap \mathrm{KEY}=K$. 因此, 由 $K \cup\{a\} \mid-\rho(m, k)$ 得 $K \cup\{a\} \mid-\left\{\rho(m, k), k^{-1}\right\}$, 故 $K \cup\{a\} \mid-m$, 注意到 $\{m\}$ 是 $m$ 的一个子项完全集, 结论 成立.

2) 若 $T$ 的根节点关联的规则是一个合成规则 $(\bullet, \rho, \mu, \operatorname{Hash}$ 或 MAC), 可用类 似的方法证明, 篇幅所限省略其过程.

综上所述, 无论根节点关联哪一条规则, 结论成立.

定义 2.1.7 $A$ 是一个消息集, 若不存在消解规则 $R\left(\in\left\{\pi_{1}, \pi_{2}, \rho_{1}, \rho_{2}, \mu^{-1}\right\}\right.$ 和消 息项 $b$ 使 $C \mid-{ }_{R} b, C$ 是 $A$ 的一个有限子集，则称 $A$ 是一个不可缩减集.

推论 2.1.1 设 $A$ 是不可缩减集, 那么, 对任何消息项 $m, m \in \mathscr{L}(A)$ 当且仅当存 在 $m$ 的子项完全集 $U_{m}$, 使得 $U_{m} \subseteq A$.

上面定理给出一个有效算法判断任一个消息项是否可由一个有限消息集 导出.

\section{2 泛多项式方程及其求解算法}

设 $A$ 是消息项集, 令 $\phi(A)=\{\phi(a) \mid a \in A\}$, 其中 $\phi=\left\{t_{1} / x_{1}, \cdots, t_{n} / x_{n}\right\}$ 是一个变量 替换.

定义 2.2.1 设 $t$ 和 $t_{1}, t_{2}, \cdots, t_{n}$ 是一些消息项, 形如 $t=\mathrm{Eq}\left(t_{1}, t_{2}, \cdots, t_{n}\right)$ 的式子称 为一个泛多项式方程. 令 $A=\left\{t_{1}, t_{2}, \cdots, t_{n}\right\}$ 是有限集, 泛多项式方程也可方便地记 作 $t=\mathrm{Eq}(A)$.

定义 2.2.2 设 $\phi$ 是一个变量替换, 若 $\phi(t) \in \mathrm{G}\left(\phi\left(t_{1}\right), \phi\left(t_{2}\right), \cdots, \phi\left(t_{n}\right)\right)$, 则称 $\phi$ 是 泛多项式方程 $t=\mathrm{Eq}\left(t_{1}, t_{2}, \cdots, t_{n}\right)$ 的一个解; 泛多项式方程 $t=\mathrm{Eq}(A)$ 的所有解的集合 称为解空间, 记作 $W_{A}(t)$.

定义 2.2.3 设 $\Phi$ 是方程 $t=\mathrm{Eq}(A)$ 的一些解的集合, 若满足对任意 $\sigma \in W_{A}(t)$, 都存在 $\phi \in \Phi$ 和变量替换 $\theta$ 使得 $\sigma=\theta \circ \phi$, 则称 $\Phi$ 为方程 $t=\mathrm{Eq}(A)$ 的一组通解.

一组通解可理解为解空间的生成元集, 那么, 这就引出了代数学中的一个 核心问题：如何计算泛多项式方程的通解? 一般情况下，这个问题是非常困 难的.

定理 2.2.1 设 $A$ 是有限消息项集, $t$ 是任一个消息项, $t=\mathrm{Eq}(A)$ 是一个泛多项式 方程. 令集合

$$
\Psi_{A}(t)=\bigcup_{U \in S(t)} \Phi_{A}(U),
$$

其中 $S(t)=\{U \mid U$ 是 $t$ 的子项完全集 $\}, \Phi_{A}(U)=\{\phi \mid$ 对任何 $u \in U$ 存在 $a \in A$ 使 $\phi(u) \unlhd_{K} \phi(a)$, 其中 $\left.K=\mathscr{L}(\phi(A)) \cap K\right\}$. 那么

1) $\Psi_{A}(t)$ 是泛多项式方程的解集, 即 $\Psi_{A}(t) \subseteq W_{A}(t)$;

2) 若在 $t$ 中仅出现 PRIM 型变量, 则 $\Psi_{A}(t)=W_{A}(t)$. 
证 1)首先, 由对 $\forall \phi \in \Psi_{A}(t)$, 由定义存在 $t$ 的一个子项完全集 $U$ 使 $\phi \in \Phi_{A}(U)$, 即对任何 $u \in U$ 有 $a \in A$ 满足 $\phi(u) \unlhd_{K} \phi(a)$, 其中 $K=\mathscr{L}(\phi(A)) \cap K$. 那么由定理 2.1.1 得 $\phi(t) \in \mathscr{L}(\phi(A))$, 从而 $\phi$ 是泛多项式方程 $t=\mathrm{Eq}(A)$ 的一个解.

2) 另一方面, 若 $\sigma$ 是方程 $t=\mathrm{Eq}(A)$ 的一个解, 即 $\sigma(t) \in \mathscr{L}(\sigma(A))$, 那么由定理 2.1.1, 存在 $\sigma(t)$ 的一个子项完全集 $U^{\prime}$ 使 $U^{\prime}$ 内包含于 $\sigma(A)$. 注意到 $t$ 中的变量只有 PRIM 型变量, 容易证明 $\sigma(t)$ 的子项完全集具有形式 $U^{\prime}=\sigma(U)$, 其中 $U$ 是 $t$ 的一个 子项完全集, 故对任何 $u \in U$ 存在 $a \in A$ 使 $\sigma(u) \unlhd_{K} \sigma(a)$, 从而 $\sigma \in \Phi_{A}(U) \subseteq \Psi_{A}(t)$, 因 此 $\Psi_{A}(t)$ 是方程的解空间.

推论 2.2.1 设 $A$ 是有限消息项集, $t$ 是仅含 PRIM 型变量的消息项, 那么, 存 在算法求解泛多项式方程 $t=\mathrm{Eq}(A)$ 的一组通解.

证 首先, 令 $U=\left\{u_{1}, \cdots, u_{m}\right\}$ 是一些项的集合, 计算集合(算法见附录 A) $\Sigma_{A}(U)=\left\{\phi_{m} \circ \phi_{m-1} \circ \ldots \circ \phi_{1} \mid\right.$ 存在 $\left\{a_{1}, \cdots, a_{m}\right\} \subseteq A$ 使得对所有的 $i=2, \cdots, m, \phi_{i}$ 是 $\phi_{i-1} \circ \cdots \circ \phi_{1}\left(u_{i}\right)$ 与 $\phi_{i-1} \circ \ldots \circ \phi_{1}\left(a_{i}\right)$ 的一个 $\left.\unlhd-M G U\right\}$.

令 $S(t)$ 是 $t$ 的所有子项完全集的集合, 下面我们证明 $U_{U \in S(t)} \Sigma_{A}(U)$ 是泛多项 式方程 $t=\mathrm{Eq}(A)$ 的一组通解.

由定理 2.2.1 直接验证 $\Sigma_{A}(U)$ 中每个元都是方程的解.

假设 $\sigma$ 是方程 $t=\mathrm{Eq}(A)$ 的任一个解, 由定理 2.2.1 存在 $t$ 的一个子项完全集 $U$ 使 $\sigma \in \Phi_{A}(U)$, 令 $U=\left\{u_{1}, \cdots, u_{m}\right\}$, 即存在 $\left\{a_{1}, \cdots, a_{m}\right\} \subseteq A$ 使得 $\sigma\left(u_{i}\right) \unlhd_{K} \sigma\left(a_{i}\right)(i=1, \cdots$, $m), K=\mathscr{L}(\sigma(A)) \cap \mathrm{KEY}$.

因 $\sigma\left(u_{1}\right) \unlhd \sigma\left(a_{1}\right)$, 那么存在 $u_{1}$ 和 $a_{1}$ 的一个 $\unlhd-\mathrm{MGU} \phi_{1}$, 使得 $\sigma=\sigma_{1} \circ \phi_{1}$.

由 $\sigma\left(u_{2}\right) \unlhd \sigma\left(a_{2}\right)$ 得到 $\sigma_{1}\left(\phi_{1}\left(u_{2}\right)\right) \unlhd \sigma_{1}\left(\phi_{1}\left(a_{2}\right)\right)$. 类似地, 存在 $\phi_{1}\left(u_{2}\right)$ 和 $\phi_{1}\left(a_{2}\right)$ 的一 个 $\unlhd-\mathrm{MGU} \phi_{2}$ 使得 $\sigma_{1}=\sigma_{2} \circ \phi_{2}$, 故 $\sigma=\sigma_{2} \circ \phi_{2} \circ \phi_{1}$, 继续下去得 $\sigma=$ $\sigma_{m} \circ \phi_{m} \circ \cdots \circ \phi_{1}$. 令 $\phi=\phi_{m} \circ \cdots \circ \phi_{1}$, 即 $\sigma=\sigma_{m} \circ \phi$, 其中 $\phi \in \Sigma_{A}(U)$.

这样证明了 $\bigcup_{U \in S(t)} \Sigma_{A}(U)$ 是泛多项式方程的一组通解.

根据上面证明过程, 得到求泛多项式方程 $t=\mathrm{Eq}(A)$ 通解的算法, 即计算下面 集合

$$
\Phi_{A}(t)=\left\{\left(\phi,\left\{a_{1}, \cdots, a_{m}\right\}\right)\left|\phi\left\{a_{1}, \cdots, a_{m}\right\} \cup K\right|-\phi(t), K=\mathscr{L}(\phi(A)) \cap K\right\} .
$$

\section{3 密码协议安全性分析算法}

\section{1 基本分析算法}

现在给出协议安全性分析过程, 基本思想是: 从安全性目标公式出发,扩张目 标公式的迹序列直到它成为正规序列, 检验是否存在不安全的序列, 从而判断协 
议的安全性. 为了方便算法描述, 我们规定一些术语.

定义 3.1.1一个状态是一个三元组 $S=(A, B, E)$, 其中 $A$ 和 $B$ 是迹序列, $E$ 是 $A$ 的正合节点集的一个子集; 设 $\Sigma$ 是一个正则迹, 若有变量替换 $\phi$ 使 $\phi(\Sigma) \sqsubseteq A$, 则 称 $S=(A, B, E)$ 是一个 $\Sigma$-状态，在上下文含义无歧义时也简称为状态.

定义 3.1.2 设 $S=(A, B, E)$ 是一个 $\Sigma$-状态, 若满足: 对所有正合 $\Sigma$-序列 $C$ 和基 本替换 $\phi$, 当 $\phi(A) \sqsubseteq C$, 有 $\phi(B) \subseteq A$. 则称 $S$ 是一个安全的 $\Sigma$-状态, 记作 $\operatorname{Secure}(S)$.

定义 3.1.3 设 $S=(A, B, E)$ 是一个 $\Sigma$-状态, 若不存在正合 $\Sigma$-序列 $C$ 和变量替换 $\phi$ 使得, $\phi(A) \sqsubseteq C$, 则称 $S$ 是一个不可达 $\Sigma$-状态.

根据定义, 不可达 $\Sigma$-状态是一个安全的 $\Sigma$-状态.

定义 3.1.4(状态扩张函数) 状态扩张函数是一个状态到一个状态集的映射, 即 $f$ 是一个状态扩张函数, $S$ 是一个状态, 那么 $f(S)=\left\{S_{1}, \cdots, S_{n}\right\}$ 是一些状态的集合 (规定 $f(S)$ 也可为空集).

定义 3.1.5 设 $f$ 是一个状态扩张函数, $S=(A, B, E)$ 是一个 $\Sigma$-状态, 若满足下 列条件, 则称 $f$ 是完备的状态扩张函数.

1) 若 $f(S)=\left\{S_{1}, \cdots, S_{n}\right\}$ 非空, 其中 $S_{i}=\left(A_{i}, B_{i}, E_{i}\right)(1 \leq i \leq n)$, 则存在变量替换 $\phi_{i}$, 使 $\phi_{i}(A) \sqsubseteq A_{i}, \phi_{i}(B)=B_{i}$; 并且

2) 对任何正合 $\Sigma$-序列 $C$ 和基本替换 $\phi$, 若 $\phi(A) \sqsubseteq C$, 则存在某个 $S_{i}=\left(A_{i}, B_{i}\right.$, $\left.E_{i}\right) \in f(S)$ 和 $\psi$, 使得 $\phi(A)=\psi \circ \phi_{i}(A)$ 且 $\psi\left(A_{i}\right) \sqsubseteq C$.

由定义 3.1 .5 条件 2$)$, 若 $f$ 是完备的状态扩张函数, 当 $f(S)$ 为空集, 那么 $S$ 是 不可达状态.

引理 3.1.1 设 $f$ 是一个完备的状态扩张函数, $S$ 是一个 $\Sigma$-状态. 令 $f(S)=\left\{S_{1}\right.$, $\left.\cdots, S_{n}\right\}$, 则 $S$ 是安全的 $\Sigma$-状态当且仅当每个 $S_{i}$ 是安全的 $\Sigma$-状态.

证 证明过程详见附录 A.

下面我们构造一个证明系统和相应的算法, 用于判断密码协议的一个安全 目标是否被模型满足, 而对于不正确的安全目标构造出相应的正合序列(攻击 过程).

先定义几个状态谓词, 设 $S=(A, B, E)$ 是一个 $\Sigma$-状态, 规定

- $\operatorname{Normal}(S)=T \Leftrightarrow B \subseteq A$, 当 $\operatorname{Normal}(S)=T$, 称 $S$ 为一个正常状态;

- Unreachable $(S)=T \Leftrightarrow S$ 是一个不可达状态;

- $\operatorname{Odd}(S)=T \Leftrightarrow B \not \subset A \wedge A \subseteq E$, 当 $\operatorname{Odd}(S)=T$, 称 $S$ 为一个奇异状态.

正常状态和不可达状态都是安全的状态, 而奇异状态是一个不安全状态. 显 然, Normal 和 Odd 均是可计算谓词. 设 $f$ 是完备的状态扩张函数, 则 $f(S)=\varnothing \Rightarrow$ Unreachable $(S)=T$. 因此, 若存在可计算的完全状态扩张函数, 则不可达 状态谓词 Unreachable 也是可计算的. 
协议安全性目标 $\Sigma$ Corresp. $\Lambda$ 和 $\Sigma$ Secret $t$ 可写成状态符号. 为此, 引入一个 仅用于安全目标中的特殊节点符号: $\langle\varepsilon, t, \Delta>$, 其中 $t$ 是消息项, $\varepsilon$ 是特定节点标号, 规定对任何节点 $\left\langle\sigma_{i j}(\alpha, l), s, s^{\prime}\right\rangle \in \Sigma_{P}$ 均有 $\left\langle\sigma_{i j}(\alpha, l), s, s^{\prime}\right\rangle \preceq_{\mathrm{P}}<\varepsilon, t, \Delta>$. 引入符号 $\Theta$ 表 示一个特殊节点, 称为奇异点, $\Theta$ 不用于构成迹序列. 规定对任何迹序列 $A$ 有 $\Theta \notin A$.

设 $A, B$ 是两个事件序列, $A \| B$ 表示 $A$ 与 $B$ 按顺序连接而成的序列. 显然, 若 $A, B$ 是迹序列且 $B$ 中没有节点 $\preceq_{P}$-先于 $A$ 中某个节点, 那么 $A \| B$ 也是迹序列.

那么, 对应性安全性目标 $\Sigma$ Corresp $\Lambda$ 用状态 $(\Sigma, \Lambda, \varnothing)$ 表示; 而秘密性安全目 标 $\Sigma$ Secret $t$ 则用 $(\Sigma, \Theta, \varnothing)$ 表示, 其中 $\Sigma=\Sigma \|<\varepsilon, t, \Delta>$.

下面给出证明系统的推理规则:

初始化规则: $\frac{(\Sigma, \Lambda, \varnothing)}{\Sigma \operatorname{Corresp} \Lambda} ; \frac{\left(\Sigma^{\prime}, \Theta, \varnothing\right)}{\Sigma \text { Secret } m}$, 其中 $\Sigma^{\prime}=\Sigma \|<\varepsilon, m, \Delta>$.

状态判定规则: $\frac{\operatorname{Secure}(S)}{\operatorname{Normal}(S)}$ Normal规则; $\frac{\operatorname{Secure}(S)}{\operatorname{Unreachable}(S)}$ Unreachable规则; $\frac{\neg \operatorname{Secure}(S)}{\operatorname{Odd}(S)} \operatorname{Odd}$ 规则.

状态扩张规则: $\frac{\left(A_{1}, B_{1}, E_{1}\right), \cdots,\left(A_{n}, B_{n}, E_{n}\right)}{(A, B, E)}$ Extend 规则, 其中 $f((A, B, E))=$ $\left\{\left(A_{1}, B_{1}, E_{1}\right), \cdots,\left(A_{n}, B_{n}, E_{n}\right)\right\}, f$ 是一个完备的状态扩张函数.

安全性目标证明过程. 为了证明协议的安全目标的正确性, 我们利用上述的 推理规则, 构造证明树. 本质上是构造攻击过程, 并通过完备状态扩张函数约束 序列产生数量, 称为约束构造算法, 简称 BCA(bounded constructing algorithm), 过程如下:

1) 对协议的安全目标运用初始化规则, 将安全目标公式转化为一个初始状 态 $S_{0}$, 并令 $Q=\left\{S_{0}\right\}$ 是一个状态队列;

2) 若 $Q$ 为空, 则算法结束, 安全目标正确; 否则, 选取 $Q$ 中的第 1 个状态 $S$;

3) 对状态 $S$ 应用 Normal 规则, 若 $\operatorname{Normal}(S)=T$, 则令 $Q=Q \backslash\{S\}$, 转执行 2); 否则执行下一步;

4) 对状态 $S$ 应用 Odd 规则, 若 $\operatorname{Odd}(S)=T$, 则算法结束, 输出相应正合序列 (攻击过程); 否则执行下一步;

5) 对状态 $S$ 进行不可达检测, 若 Unreachable $(S)=T$, 则令 $Q=Q \backslash\{S\}$, 转执行 2); 否则执行下一步;

6) 对 $S$ 执行状态扩张规则, 令 $f(S)=\left\{S_{1}, \cdots, S_{n}\right\}$, 并将 $\left\{S_{1}, \cdots, S_{n}\right\}$ 置于队列 $Q$ 之后, 即 $Q=Q \cup\left\{S_{1}, \cdots, S_{n}\right\}$, 转 2). 
容易看到算法只能在第 2)步或第 4)步中结束, 若算法在第 2)步结束, 那么安 全目标是正确的; 若安全目标是不正确的, 那么算法会在第 4)步结束. 算法过程 中除第 6)步外，其他步骤使总状态数减少，因此，算法是否会停止取决于第 6)步

状态扩张.

算法可靠性.

引理 3.1.2 1) 设 $\Sigma$ Corresp $\Lambda$ 是密码协议 $P$ 的一个安全目标, 则 $P$ 满足 $\Sigma$ Corresp $\Lambda$ 当且仅当 $(\Sigma, \Lambda, \varnothing)$ 是一个安全的 $\Sigma$-状态.

2) 设 $\Sigma$ Secret $m$ 是密码协议 $P$ 的一个安全目标, 则 $P$ 满足 $\Sigma$ Secret $m$ 当且仅 当 $(\Sigma, \Theta, \varnothing)$ 是一个安全的 $\Sigma$-状态, 其中 $\Sigma=\Sigma \mid<\varepsilon, m, \Delta>$.

证 由定义直接可证.

引理 3.1.3 设 $\Sigma$ Corresp $\Lambda$ (或 $\Sigma$ Secret $m$ ) 是密码协议 $P$ 的一个安全性目标, 若在经 BCA 过程, 以安全状态停止, 则 $\Sigma$ Corresp $\Lambda$ (或 $\Sigma$ Secret $m$ ) 是正确的.

证 如果经 BCA 以安全状态停止, 则存在以安全目标为根节点的一棵证明 树, 而且每个叶节点均为安全状态.

注意到每个节点状态 $S$ 的所有儿子节点集为 $f(S), f$ 是完备状态扩张函数, 那 么由引理 3.1.1 和引理 3.1.2一个节点是安全状态当且仅当它的所有儿子节点是安 全状态. 因此, 根节点表示的安全目标是正确的当且仅当证明树的每个叶节点的 状态是安全的.

若算法停止, 则证明树的每个叶节点必然是正常状态、或不可达状态或奇异 状态中一种. 因此, 可容易地判断叶节点状态的安全性, 从而可判断安全性目标 的正确性.

\section{2 基于泛多项式方程求解的完备状态扩张函数}

现在构造完备的状态扩张函数, 由基本算法 $\mathrm{BCA}$ 过程看到, 要使算法尽快 停止, 关键在于构造一个尽可能产生较少状态数的完备状态扩张函数, 这里我们 利用泛多项式方程求解算法, 来约束状态数的产生量, 从而提高算法效率.

为了简化算法的描述, 先规定一些术语和符号：设 $\left\langle\sigma_{i k}(\alpha, l), s_{1}, t_{1}\right\rangle$ 和 $\left\langle\sigma_{i j}(\alpha, l), s_{2}, t_{2}\right\rangle$ 是两个节点, 若 $k<j$, 我们称 $\left\langle\sigma_{i k}(\alpha, l), s_{1}, t_{1}\right\rangle$ 是 $\left\langle\sigma_{i j}(\alpha, l), s_{2}, t_{2}\right\rangle$ 的一 个前驱节点.

定义 3.2.1 设 $B$ 是节点集, 若 $B$ 的每个节点的前驱节点也属于 $B$, 则称 $B$ 是 一个前驱封闭集. 设 $C$ 是一些主节点集, 而 $B$ 是包含 $C$ 的最小前驱封闭集, 则称 $B$ 是 $C$ 的一个前驱闭包.

定义 3.2.2 设 $\left\langle\sigma_{i j}(\alpha, l), s, t\right\rangle$ 是一个事件, $t$ 称为节点 $\left\langle\sigma_{i j}(\alpha, l), s, t\right\rangle$ 的输出项, 或称 $t$ 源于节点 $\left\langle\sigma_{i j}(\alpha, l), s, t\right\rangle$, 记作 $t \leftarrow\left\langle\sigma_{i j}(\alpha, l), s, t\right\rangle$. 设 $\Gamma$ 是节点集, 而 $A$ 是 $\Gamma$ 的 
输出项的集合, 称 $A$ 源于 $\Gamma$, 记作 $A \leftarrow \Gamma$; 若对 $A$ 中任一个项 $t$, 在 $\Gamma$ 中只有唯一节 点的输出项为 $t$, 则称 $A$ 对等于 $\Gamma$, 记作 $A \leftrightarrow \Gamma$.

设 $C=\left\langle w_{1}, s_{1}, s_{1}^{\prime}\right\rangle\left\langle w_{2}, s_{2}, s_{2}^{\prime}\right\rangle \cdots\left\langle w_{n}, s_{n}, s_{n}^{\prime}\right\rangle$ 是一个节点序列, 符号 $[C, k]$ 表示 $C$ 的第 $k$ 个节点 $\left\langle w_{k}, s_{k}, s_{k}^{\prime}\right\rangle$, 并用 $\left[C, i_{1}, i_{2}, \cdots, i_{m}\right]$ 表示节点序列 $\left[C, i_{1}\right]\left[C, i_{2}\right] \cdots[C$, $\left.i_{m}\right]$, 其中 $\left\{i_{1}, i_{2}, \cdots, i_{m}\right\} \subseteq\{1,2, \cdots, n\}$

定义 3.2.3 设 $G$ 是一些节点集合, 取 $G$ 中所有节点的一个排列 $C$, 如果这个 节点序列的先后顺序不违背序关系 $\preceq_{\mathrm{P}}$, 即当 $k>j$ 不存在 $[C, k] \preceq_{\mathrm{P}}[C, j]$, 则称 $C$ 是 $G$ 的一个有效排列.

定义 3.2.4 设 $A$ 是长度为 $m$ 的节点序列, $\left\langle w, r, r^{\prime}\right\rangle$ 是一个节点, 设 $A^{\prime}$ 是按下 面方法构造的节点序列, 我们称 $A^{\prime}$ 是将 $\left\langle w, r, r^{\prime}\right\rangle$ “靠后优先”插入 $A$ 得到的节点序 列, 其中 $\phi\left(\left\langle w, r, r^{\prime}\right\rangle\right) \varliminf_{P} \phi([A, j])$ 表示 $\neg \phi\left(\left\langle w, r, r^{\prime}\right\rangle\right) \preceq_{P} \phi([A, j])$.

1) 若 $\left\langle w, r, r^{\prime}\right\rangle \preceq_{\mathrm{P}}[A, 1]$, 则 $A^{\prime}=\left\langle w, r, r^{\prime}\right\rangle \| A$ ；

2) 若对所有 $1 \leq j \leq m$ 有 $\left\langle w, r, r^{\prime}\right\rangle \underline{\mathrm{N}}_{\mathrm{P}}[A, j]$, 则 $A^{\prime}=A \|\left\langle w, r, r^{\prime}\right\rangle$;

$3)$ 若存在 $1<j \leq m$ 使 $\left\langle w, r, r^{\prime}\right\rangle \preceq_{P}[A, j]$, 并且对 $\forall k<j,\left\langle w, r, r^{\prime}\right\rangle \underline{\aleph}_{P}[A, k]$, 则令 $A^{\prime}=[A, 1, \cdots, j-1]\left\|\left\langle w, r, r^{\prime}\right\rangle\right\|[A, j, \cdots, m]$.

正合扩张算法. 诚实主体会话 $(\alpha, l), \mathrm{NOR}_{P}$ 是相应的主节点集, 令 $D_{P}$ 是所有 主节点输出消息项集, 即

$$
D_{P}=\left\{t \mid\langle\sigma, s, t\rangle \in \mathrm{NOR}_{P}\right\} .
$$

令 $D=D_{P} \cup D_{0}$, 其中 $D_{0}$ 是攻击者的初始知识.

设 $S=(A, B, E)$ 是一个 $\Sigma$-状态, 其中 $A=\left\langle w_{1}, s_{1}, s_{1}^{\prime}\right\rangle\left\langle w_{2}, s_{2}, s_{2}^{\prime}\right\rangle \cdots\left\langle w_{n}, s_{n}, s_{n}^{\prime}\right\rangle$, 我 们来构造一个完备的状态扩张函数 $f$.

算法开始: 当 $A \subseteq E, S$ 或者是正常状态或者奇异状态, 这时我们规定 $f(S)=\{S\}$; 否则, 选取 $A$ 的第一个不属于 $E$ 的节点(非正合节点) $[A, m]=\left\langle w_{m}, s_{m}, s_{m}^{\prime}\right\rangle \notin E(1 \leq m$ $\leq n)$. 分两种情形:

1) 若 $s_{m}=\Delta$ 或 $D_{0} \cup\left\{s_{1}, \cdots, s_{m-1}\right\} \mid-s_{m}$, 那么规定 $f(S)=\left\{\left(A, B, E \cup\left\{\left\langle w_{m}, s_{m}, s_{m}^{\prime}\right\rangle\right\}\right)\right\}$.

2) 否则, 求解泛多项式方程 $s_{m}=\mathrm{Eq}(D)$ 的通解 $\Phi_{D}\left(s_{m}\right)=\left\{\left(\phi, t_{1}, \cdots, t_{k}\right) \mid t_{i} \in D\right\}$, 这 是一个有限集. 执行 $1^{\circ}, 2^{\circ}, 3^{\circ}$ 步骤.

$1^{\circ}$ 若 $\Phi_{D}\left(s_{m}\right)=\varnothing$, 则规定 $f(S)=\varnothing$.

$2^{\circ}$ 若 $\Phi_{D}\left(s_{m}\right) \neq \varnothing$, 则对每个 $\left(\phi, t_{1}, \cdots, t_{k}\right) \in \Phi_{D}\left(s_{m}\right)$, 执行下列(a), (b) (c)步骤:

(a) 若所有 $t_{i} \in D_{0}(1 \leq i \leq k)$, 则得到一个新状态 $S_{\phi}=(\phi(A), \phi(B), \phi(E \cup$ $\left.\left.\left\{\left\langle w_{m}, s_{m}, s_{m}^{\prime}\right\rangle\right\}\right)\right)$;

(b) 否则, 令 $T=\left\{t_{1}, \cdots, t_{k}\right\} \backslash D_{0}$, 求集合 $D_{T}=\left\{\Sigma_{T} \subseteq \mathrm{NOR}_{P} \mid T \leftrightarrow \Sigma_{T}\right\}$, 这是有限集. 对每个 $\Sigma_{T} \in D_{T}$, 执行以下 i, ii 步骤: 
i ) 若存在 $\langle\Gamma, r, t\rangle \in \Sigma_{T}$ 和 $A$ 的一个节点 $[A, j](j \geq m)$ 使 $\phi([A, j]) \preceq_{\mathrm{P}} \phi(\langle\Gamma, r, t\rangle)$ ， 则规定 $S_{\Sigma_{T}}=\varnothing$. 否则执行 ii .

ii ) 求集合 $\Sigma_{T}$ 的前驱闭包 $\bar{\Sigma}_{T}$, 令

$H_{0}=\left\{\left\langle w, r, r^{\prime}\right\rangle \in \bar{\Sigma}_{T} \mid\right.$ 存在 $j<m$, 使 $\phi(w)=\phi\left(w_{j}\right)$ 且 $\phi(r) \neq \phi\left(\mathrm{s}_{j}\right) \vee \phi\left(r^{\prime}\right)=\phi\left(s_{j}^{\prime}\right)$. 执行下面(1), (2)步骤:

(1) 若 $H_{0} \neq \varnothing$, 并且不存在变量替换 $\psi$ 使 $\psi \phi\left(H_{0}\right) \subseteq \psi \phi(A)$, 则不产生新的状态, $S_{\Sigma_{T}}=\varnothing$, 否则执行(2).

(2) 假设存在变量替换 $\psi$ 使 $\psi \phi\left(H_{0}\right) \subseteq \psi \phi(A)$ (当 $H_{0}=\varnothing$, 则规定 $\psi$ 为空替换), 令 $H=\psi \phi\left(\bar{\Sigma}_{T} \backslash H_{0}\right), G=\{C \mid C$ 是 $H$ 的一个有效全排列 $\}, A_{0}=\psi \phi([A, 1, \cdots, m-1])$ 是 $\psi \phi(A)$ 子迹序列.

选取 $C \in G$, 将节点 $[C, 1]$ 按“靠后优先”插入 $A_{0}$ 得到节点序列 $A_{1}$; 将节点 $[C, 2]$ 按“靠后优先”插入 $A_{1}$ 得到节点序列 $A_{2}$; 继续下去, 最后得到节点序列 $A_{C}$.

令 $A_{C}^{\prime}=A_{C} \| \psi \phi([A, m, \cdots, n])$, 得到一个新的状态

$$
S_{C}^{\prime}=\left(A_{C}^{\prime}, \psi \phi(B), \psi \phi\left(E \cup\left\langle w_{m}, s_{m}, s_{m}^{\prime}\right\rangle\right)\right),
$$

那么, 对所有 $C \in G$ 得到状态集 $S_{\Sigma_{T}}=\left\{S_{C}^{\prime} \mid C \in G\right\}$.

(c) 令 $S_{\phi}=\cup_{\Sigma_{T} \in D_{T}} S_{\Sigma_{T}}$, 它是根据 $\phi$ 扩张的状态集.

$3^{\circ}$ 最后, 令 $f(S)=\bigcup_{\phi \in \Phi_{D}\left(s_{m}\right)} S_{\phi}$.

算法结束.

这样我们完成了对状态 $S$ 的扩张过程. 实质上, 上述过程是对安全目标中的 迹序列 $A$ 进行“正合扩张”过程.

引理 3.2.1 设 $S=(A, B, E)$ 是由安全目标在上面扩张算法过程中产生的一个 $\Sigma$-状态, 而 $\Lambda$ 是一个正合的迹实例, 若存在基本变量替换 $\eta$ 使 $\eta(A) \sqsubseteq \Lambda$, 那么存在 基本变量替换 $\psi$ 和由主节点组成的迹序列 $\Gamma$ 使 $\Lambda=\psi(\Gamma)$ 且 $\eta(A)=\psi(A)$.

限于篇幅省略其详细证明.

定理 3.2.1 由上面描述的扩张算法所定义的 $f$ 是一个完备的状态扩张函数.

证 假设 $\Lambda$ 是一个正规 $\Sigma$-序列, $S=(A, B, E)$ 是一个 $\Sigma$-状态, 若存在基本变量替 换 $\eta$ 使 $\eta(A) \sqsubseteq \Lambda$, 需要证明存在变量替换 $\phi, \varphi$ 和 $S^{\prime}=\left(A^{\prime}, B^{\prime}, E^{\prime}\right) \in f(S)$, 使 $\varphi\left(A^{\prime}\right) \sqsubseteq \Lambda$ 且 $\eta=\varphi \circ \phi$.

令 $[A, m]=\left\langle w_{m}, s_{m}, s_{m}^{\prime}\right\rangle$ 是 $A$ 的第一个不属于 $E$ 的节点(注: 若 $A \subseteq E$, 结论是平 凡的), 设 $\eta\left(\left\langle w_{m}, s_{m}, s_{m}^{\prime}\right\rangle\right)=[\Lambda, p]$, 由引理 3.2.1, 存在基本变量替换 $\psi$ 和由主节点 组成的迹序列 $\Gamma$ 使 $\Lambda=\psi(\Gamma)$ 且 $\eta(A)=\psi(A)$. 又由 $\Lambda$ 的正合性条件得到 


$$
D_{0} \cup\left\{\psi\left(t_{1}\right), \cdots, \psi\left(t_{k}\right)\right\} \mid-\psi\left(s_{m}\right),
$$

其中 $\left\{t_{1}, \cdots, t_{k}\right\} \leftarrow\{[\Gamma, 1], \cdots,[\Gamma, p-1]\},\{[\Gamma, 1], \cdots,[\Gamma, p-1]\} \subseteq \mathrm{NOR}_{P}$, 那么 $\left(\psi, t_{1}\right.$, $\left.\cdots, t_{k}\right)$ 是泛多项式方程 $s_{m}=\mathrm{Eq}(D)$ 的一个解, 因此存在 $\phi \in \Phi_{D}\left(s_{m}\right)$ 和 $\varphi$ 使 $\psi=\varphi \circ \phi$.

那么, 由 $f$ 的算法过程可知: 存在 $S^{\prime}=\left(A^{\prime}, B^{\prime}, E^{\prime}\right) \in f(S)$, 其中 $B^{\prime}=\phi(B)$, 且 $A^{\prime}$ 的每 个节点 $\left[A^{\prime}, i\right] \in \phi(A)$ 或 $\left[A^{\prime}, i\right] \in \phi\left(\Sigma_{Q}\right)$. 这里 $Q=\{[\Gamma, 1], \cdots,[\Gamma, p-1]\}$ ，因此，得到

$$
\varphi\left(\left[A^{\prime}, i\right]\right) \in \varphi(\phi(A))=\psi(A) \text { 或 }\left[A^{\prime}, i\right] \in \psi\left(\Sigma_{Q}\right) \text {. }
$$

但 $\psi(A)=\eta(A) \sqsubseteq \Lambda, \psi\left(\Sigma_{Q}\right) \subseteq \Lambda$, 故 $\varphi\left(A^{\prime}\right) \sqsubseteq \Lambda$ 且 $\eta(A)=\varphi \circ \phi(A)$, 满足定义 3.1.5 的条 件 2).

\section{3 算法完备性和复杂性讨论}

完备性讨论. 前面已阐述用BCA过程验证一个安全性目标算法停止, 那么可 以判断该安全性目标是否正确. 若算法完成证明过程, 以安全状态结束, 则该公 式是正确的; 若算法检测到攻击序列, 则可判定协议是不安全的. 然而, 算法是 否一定会停止呢? 答案是否定的. 从算法过程看: 由于 $\mathrm{NOR}_{P}$ 中有ID变量(表示攻 击者)和自然数变量, 可能造成无限并发和串行, 状态扩张算法并不能保证扩张 的状态数最终会变少, 因此, 也不能保证算法一定会停止. 事实上, 已证明即便 在更为严格条件限制下, 密码协议的安全性仍是不可判定的 ${ }^{[7]}$. 然而, 我们有下 面结论.

定义 3.3.1 $f$ 是协议 $P$ 的完备状态扩张函数, 若存在无限状态序列

$$
S_{1} \rightarrow S_{2} \rightarrow \cdots \rightarrow S_{n} \rightarrow \cdots,
$$

其中 $S_{i+1} \in f\left(S_{i}\right)$, 则称 $f$ 是发散的完备状态扩张函数; 否则称 $f$ 是收玫的.

定理 3.3.1 $P$ 是一个密码协议, 若存在一个收玫的完备状态扩张函数, 那么 密码协议 $P$ 的安全性是可判定的.

复杂性讨论. 由上所述, 针对一般性密码协议而言, BCA 过程不能保证一定 会停止. 当然也不能保证多项式时间内的复杂度, 至于哪些类型协议可在多项式 时间内完成分析，则是另一个困难的问题. 然而，对于一些实际的协议 BCA 算法 是相当高效的, 这里仅从直观角度简要讨论算法复杂性.

我们知道协议安全性分析的复杂性主要源于三个方面：参与主体大量性, 协 议的并发与交错运行和消息计算复杂性.

参与主体数量问题. 这里通过人工证明, 使协议参与主体仅包括几个诚实主 体, 用变量表示攻击者的 ID, 避免由于主体过多而引起的大量对称几余状态.

并发数量问题. 一些基于模型检测工具协议方法, 通过对称技术、部分序方 法等减少状态搜索, 而一些基于定理证明方法, 如 NRL, Athena 等通过符号和变 量表示等方法减少状态搜索. 我们主要通过下面方法来解决这个问题: 
- 用简洁的模型形式描述密码协议, 减少协议的描述几余. 例如, 仅需要 4 个基本事件函数可描述 Needham-Schroeder 公开密钥协议, 那么, 所有的攻击活 动序列可用这 4 个函数的实例序列描述, 这样减少了协议的状态空间.

- 采用泛多项方程求解来约束需要搜索的迹序列数量, 排除了分析迹过 程中产生的大量冗余状态, 加速算法的收玫.

消息计算复杂性. 一般的工具通常由于消息运算引起状态爆炸, 因此, 往往 人为限制消息的计算结构. 例如, 限制消息的加密重数, 这实质上是限制了攻击 者的消息计算能力, 从而影响了分析结果的可靠性.

我们采用代数方法解决这个问题, 通过求解代数方程约束消息项的产生数 量, 无需限制攻击者的消息运算能力, 还可使用多种密码算法, 从而提高证明结 果的可靠性和算法的适用性.

基本算法一个显著的特点是：我们没有对前文所提到的引起无限状态空间 的 4 种原因中任何一个方面进行了人为限制, 因此, 该算法(如果停止)可用于提 供协议在无限状态运行的安全性分析.

\section{4 算法优化讨论}

参数限制. 由于算法可能不停止, 这时需要人工干预分析过程, 这影响了系 统的自动化程度, 也给非专业用户使用带来困难. 因此, 系统实现时可通过设置 参数, 如限制变量 ID 的匹配数量和会话参数, 这样搜索空间形成一个有限系统, 然而, 本质上, 这是一种模型检测方法, 不能证明提供协议安全性证明, 需要设 置合适的参数提高分析的可靠性.

不可达状态检测. 算法在序列扩张过程中, 特别是在证明协议的安全性时, 会有许多不可达状态, 因此, 如何及早判断状态的不可达性对于提高算法效率有 重要的意义. 对于一般性密码协议, 这看起来是一个较难解决的问题, 但对于一 些具体协议, 可利用协议的特殊性质, 及早判断不可达状态. 因此, 在算法模块 中, 允许用户插入状态不可达检测模块算法, 以使算法尽快收玫. 但是状态不可 达检测依赖于具体的密码协议特点, 必须根据具体协议由用户确定, 作为一种通 用工具, 这给非专业用户带来使用困难.

\section{4 系统实现与实验分析}

\section{1 安全协议安全性自动分析系统实现}

根据 CPA 模型和分析算法, 我们用 JAVA 语言实现了一个安全协议自动分析 系统, 称为 ACT-SPA (security protocol analyzer).

ACT-SPA 软件系统主要有三个模块: 
1) 安全协议的输入：根据 CPA 模型的描述语言，描述安全协议的消息交互 和安全目标. ACT-SPA 为用户了提供方便的图形界面和编辑器输入安全协议;

2) 证明结果显示：对协议的分析过程实现图形化显示，用户可直观地了解 协议的分析过程，如果存在安全协议的缺陷，可以构造出攻击过程;

3) 协议分析算法: 实现密码协议的安全性目标的自动分析. 若检测到攻击, 系统可输出相应攻击序列, 若未发现攻击序列; 系统提供安全目标证明过程结果 供使用者分析参考.

\section{2 实验分析}

利用SPA系统, 我们分析了二十多个密码协议的安全性, 发现所有已知的攻 击, 并发现一个我们所知文献中未提到的一个密码协议攻击(附录A中给出BANYahalom协议 ${ }^{[13]}$ 的分析示例).

对比了一些协议分析所需要搜索的状态数, 对于许多协议 ACT-SPA 一般仅 用几十个状态即发现攻击序列，较多的有几百个状态. 表 1 是与相关系统的实验 数据比较, 表中数据是系统发现攻击或完成分析的状态数, 其中 ACT-SPA 是完 成一个角色验证过程的状态数. 表中是文献中公开相关的实验数据.

表 1 与同类系统分析效率对比表

\begin{tabular}{cccccc}
\hline 协议 & Mur $\phi$ & Brutus & Athena & STA & ACT-SPA \\
\hline Needham-Schroeder 公开密钥协议 & 1706 & 1208 & 36 & $*$ & 8 \\
Needham-Schroeder-Lowe 公开密钥协议 & $*$ & 146 & 19 & 60 & 15 \\
TMN 协议 & $*$ & 3327 & $*$ & $*$ & 5 \\
Kerberos 协议(简化版) & $*$ & 3405 & $*$ & $*$ & 6 \\
\hline
\end{tabular}

表中为各个系统找到攻击或完成证明搜索的状态数, *表示我们未获得相关实验数据. Mur $\phi$ 是 Stanford 大学 1992 年研制的模型检测工具, 1997 年应用于密码协议分析; Brutus是Carnegie Mellon大学等 2000 年研 制的模型检测工具; Athena是Carnegie Mellon大学 2001 年研制的密码协议分析工具; STA是意大利Firenze大 学 2001 年研制的密码协议分析工具 $\frac{[14]}{}$

\section{5 结论}

本文的主要工作: 根据密码协议的 CPA 模型, 设计了新的密码协议安全性分 析算法. 根据 CPA 模型中的迁移函数提出了泛多项式方程的概念，并给出相应的 泛多项方程求解算法, 利用泛多项方程求解算法约束状态产生的数量, 从而建立 了一种新的密码协议安全性分析算法. 该算法具有特点:

- 采用 CPA 模型的简化密码协议的描述, 并且利用泛多项式方程求解约 束算法，更精确约束了消息项的产生，排除了分析过程中大量几余状态;

- 该算法未对引起无限状态空间的各种因素进行人为限制, 因此可提供 密码协议在无限空间运行下的安全性分析. 
实验结果表明: 根据算法研制的协议安全性分析系统显著地提高了运行效 率和可靠性.

$$
\text { 参考文献 }
$$

1 Dolev D, Yao A. On the security of public key protocols. IEEE Transactions on Information Theory, 1983, 29(2): 198 208[DOI]

2 Comon H, Shmatikov V. Is it possible to decide whether a cryptographic protocol is secure or not? Journal of Telecommunications and Information Technology, 2002 (4): 5 15

3 Lowe G. Breaking and fixing the Needham-Schroeder public-key protocols using FDR. In: Lecture Notes in Computer Science, 1055. Berlin: Springer-Verlag, 1996: 147 166

4 Mitchell J C, Mitchell M, Stern U. Automated analysis of cryptographic protocols using mur $\phi$. In: Proceedings of the 1997 IEEE Symposium on Security and Privacy. IEEE Computer Society Press, 1997

5 Millen J. The interrogator model. In: Proceedings of the 1995 IEEE Symposium on Security and Privacy. IEEE Computer Society Press, 1995. 251 260

6 Clarke E, JHA S, Marrero W. Verifing security protocols with Brutus. ACM Transactions on Software Engneering and Methodology, 2000, 9(4): 443 487[DOI]

7 Durgin N, Lin C P. Undecidability of bounded security protocols. In: Electronic Proceedings of the Workshop on Formal Methods and security protocols, 1999

8 Meadows C. A model of computation for the NRL protocol analyzer. In: Proceedings of the 1994 Computer Security Foundations Workshop. IEEE Computer Society Press, 1994

9 Song D, Berezin S, Perrig A. Athena: a novel approach to efficient automatic security protocol analysis. Journal of Computer Security, 2001, 9(2): 47 74

10 Huima A. Efficient infinite-state analysis of security protocols. In: Proceedings FLOC Workshop on Formal Methods of Security Protocols, Italy, 1999

11 Huai J P, Li X X. Algebra model and security analysis for cryptographic protocols. Science in China, Series F, 2004, 47(2): 199 220[Abstract]

12 Thayer F F, Herzog J C, Guttman J D. Honest ideals on strand spaces. In: Proceedings of 1998 Computer Security Foundations Workshop, 1998

13 Paul Syverson. A taxonomy of replay attacks. In: Proceedings of the Computer Security Foundations Workshop VII, Franconia NH, 1994, 187 191

14 Boreale M, Buscemi M. Experimenting with STA, a tool for untomatic analysis of securily Protocols. In: Proc of SAC'o2. ACM Press, 2002. 281 285

\section{附录 A}

\section{1. 计算 $\Sigma_{A}(U)$ 的算法:}

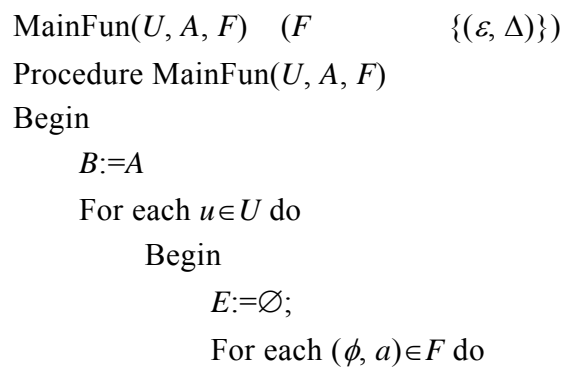




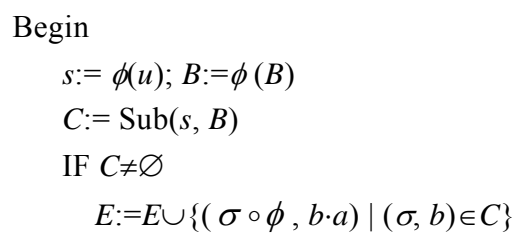

End

$$
F:=E
$$

End

Return $F$

End

$\operatorname{Sub}(s, B)=\left\{(\sigma, b) \mid\right.$ 存在 $b \in B$ 使 $\sigma$ 是 $s$ 与 $b$ 的 $\unlhd-\mathrm{MGU}$, 且 $\sigma(s) \unlhd_{K} \sigma(b)$ 其 中 $K=\mathscr{L}(\sigma(B)) \cap K\}$

注 A1 $\varepsilon$ 表示空替换, $\Delta$ 表示“空项”.

2. 引理 3.1.1 的证明.

证 “ $\Rightarrow$ ”. 对 $S_{i}=\left(A_{i}, B_{i}, E_{i}\right)$, 设有正合 $\Sigma$-序列 $C$ 和基本替换 $\psi$, 使 $\psi\left(A_{i}\right) \sqsubseteq C$, 因 $f$ 是完 备的, 由定义存在变量替换 $\phi_{i}$ 使 $\phi_{i}(A) \sqsubseteq A_{i}, \phi_{i}(B)=B_{i}$, 从而得到

$$
\psi\left(\phi_{i}(A)\right) \sqsubseteq \psi\left(A_{i}\right) \sqsubseteq C \text { 和 } \psi\left(\phi_{i}(B)\right)=\psi\left(B_{i}\right) .
$$

又因 $S$ 是安全的 $\Sigma$-状态, 得 $\left(\psi \circ \phi_{i}\right)(B) \sqsubseteq C$, 即 $\psi\left(B_{i}\right) \subseteq C$, 这证明 $S_{i}=\left(A_{i}, B_{i}, E_{i}\right)$ 是一个 安全的 $\Sigma$-状态.

“ $\Leftarrow$ ” 对任何正合 $\Sigma$-序列 $C$ 和基本替换 $\phi$, 若 $\phi(A) \sqsubseteq C$, 由 $f$ 的完备性, 存在状态 $S_{i}=\left(A_{i}, B_{i}, E_{i}\right)$ 和基本替换 $\psi$ 使得 $\phi(A)=\psi \circ \phi_{i}(A)$ 且 $\psi\left(A_{i}\right) \sqsubseteq C$. 由假设 $S_{i}$ 是安全的, 即 $\psi\left(B_{i}\right) \subseteq C$ ，但 $\phi_{i}(B)=B_{i}$, 另外注意到 $\operatorname{Var}(B) \subseteq \operatorname{Var}(A)$ 而 $\phi(A)=\psi \circ \phi_{i}(A)$, 故 $\phi(B)=\psi \circ \phi_{i}(B)$, 因此,

$$
\phi(B)=\psi\left(B_{i}\right) \subseteq C .
$$

由定义 $3.15, S$ 是一个安全的 $\Sigma$-状态.

3. 关于 BAN-Yahalom 协议分析.

BAN-Yahalom 协议是 Burrows, Abadi 和 Needham 对 Yahalom 协议的一个修改版本. 这个协议的目的是, 通信双方通过可信第三方实现相互的身份认证, 并建立一个新的会 话密钥.

BAN-Yahalom 协议在 CPA 模型中的描述:

角色 $A$ 的迹序列 $\Sigma_{1}(\alpha, l): \begin{aligned} & \left\langle\sigma_{11}(\alpha, l), \Delta,\left(A, N_{a}\right)\right\rangle, \\ & \left\langle\sigma_{12}(\alpha, l),\left(x,\left\{B, k, N_{a}\right\}_{K_{a s}},\{A, k, x\}_{K_{b s}}\right),\left(\{A, k, x)_{K_{b s}},\{x\}_{k}\right)\right\rangle ; ;\end{aligned}$

角色 $B$ 的迹序列 $\Sigma_{2}(\alpha, l): \begin{aligned} & \left\langle\sigma_{21}(\alpha, l),(A, y),\left(B, N_{b},\{A, y\}_{K_{b s}}\right)\right\rangle, \\ & \left\langle\sigma_{22}(\alpha, l),\left(\left\{A, k^{\prime}, N_{b}\right\}_{K_{b s}},\left\{N_{b}\right\}_{k^{\prime}}\right), \Delta\right\rangle ;\end{aligned}$

角色 $S$ 的迹序列 $\Sigma_{3}(\alpha, l)$ : 


$$
\left\langle\sigma_{31}(\alpha, l),\left(B, z,\{A, w\}_{K_{b s}}\right),\left(z,\left\{B, K_{a b}, w\right\}_{K_{a s}},\left\{A, K_{a b}, z\right\}_{K_{b s}}\right)\right\rangle,
$$

其中 $x, k, z, y, k^{\prime}$ 和 $w$ 是 PRIM 型消息变量, 我们用 $\{m\}_{k}$ 表示消息项 $\mu(m, k)$.

协议的对应性安全目标定理:

$$
\begin{aligned}
& \Sigma_{1}(\alpha, 1) \operatorname{Corresp}\left\langle\sigma_{21}(\alpha, 1),\left(A, N_{a}\right),\left(B, N_{b},\left\{A, N_{a}\right\}_{K_{b s}}\right)\right\rangle, \\
& \Sigma_{2}(\alpha, 1) \operatorname{Corresp} \Sigma_{1}(\alpha, 1)\left\{N_{b} / x, K_{a b} / k\right\} .
\end{aligned}
$$

ACT-SPA 在 PC 机(CPU 为 PIII450 MHz, 内存 $128 \mathrm{MB}$ )上运行分析该协议, 在不到 1 秒时间内就得到分析的结果: 角色 $A$ 的安全目标成立, 但角色 $B$ 的安全目标则不成立. 系 统给出了一个目前公开文献末见的一个攻击, 用 CPA 模型语言描述如下:

$$
\begin{aligned}
& \left\langle\sigma_{11}(\alpha, 1), \Delta,\left(A, N_{a}\right)\right\rangle \rightarrow \\
& \left\langle\sigma_{21}(\alpha, 1),\left(A, N_{a}\right),\left(B, N_{b},\left\{A, N_{a}\right\}_{K_{b s}}\right)\right\rangle \rightarrow \\
& \left\langle\sigma_{21}(\beta, 1),\left(B, N_{b}\right),\left(A, N_{a}^{\prime},\left\{B, N_{b}\right\}_{K_{a s}}\right)\right\rangle \rightarrow \\
& \left\langle\sigma_{31}(\beta, 1),\left(A, N a,\left\{B, N_{b}\right\}_{K_{a s}}\right),\left(N_{a},\left\{A, K_{a b}^{\prime}, N_{b}\right\}_{K_{b s}},\left\{B, K_{a b}^{\prime}, N_{a}\right\}_{K_{a s}}\right)\right\rangle \rightarrow \\
& \left\langle\sigma_{12}(\alpha, 1),\left(N_{b},\left\{B, K_{a b}^{\prime}, N_{a}\right\}_{K_{a s}},\left\{A, K_{a b}^{\prime}, N_{b}\right\}_{K_{b s}}\right),\left(\left\{A, K_{a b}^{\prime}, N_{b}\right\}_{K_{b s}},\left\{N_{b}\right\}_{K_{a b}^{\prime}}\right)\right\rangle \rightarrow \\
& \left\langle\sigma_{22}(\alpha, 1),\left(\left\{A, K_{a b}, N_{b}\right\}_{K_{b s}},\left\{N_{b}\right\}_{K_{a b}}\right), \Delta\right\rangle,
\end{aligned}
$$

其中 $\alpha=(A, B, S), \beta=(B, A, S)$.

在这个针对参数为 $(\alpha, 1)$ 的会话的重放攻击过程中, 攻击者冒充 $B$ 的身份发起另一个 并发的协议会话进程, 篡改了原协议会话第三步中 $S$ 发给 $A$ 的消息, 从而达到了为 $A, B$ 分 配一个旧密钥 $\left(K_{a b}^{\prime}\right)$ 的目的. 由于 $A, B$ 间的双向认证并未被破坏, 这就欺骗了 $A, B$, 使他 们误以为 $K_{a b}^{\prime}$ 是 $S$ 为本轮会话生成的新会话密钥, 从而破坏了协议的安全性目标(会话密 钥的新鲜性).

文献[13]认为 BAN-Yahalom 协议存在弱点, 但给出的攻击是在假设协议参与者不检 查随机数的情况下成立, 即假设“角色 $B$ 在第一步中不检查收到的新鲜值 $N_{a}$ 的长度”(一般 在协议的实现中会规定所用的新鲜值的比特位数), 或“ $S$ 在第二步中不对接收到的消息中 的 $N_{a}$ 和 $N_{b}$ 进行比较” (一般认为在同一次协议会话过程中所用的新鲜值应互不相同, 即 $N_{a}$ $\neq N_{b}$ ), 这是模型外的假设条件, 因此, 严格的说这还不能构成对协议本身攻击. 然而, 我 们发现的攻击过程无需模型外的假设条件, 就可成功对协议攻击, 这个攻击意味着 BAN-Yahalom 协议设计本身存在着漏洞. 